\title{
An Improved Federal Kalman Filter Based on Smoother for Wireless Localization
}

\author{
$\mathrm{Xi}$ Zhong $\mathrm{Lou}^{1}$ and Dao Rong $\mathrm{Wu}^{2}$ \\ ${ }^{1}$ Associate professor at Information \\ ${ }^{2}$ Master Student at Information, \\ ${ }^{1,2}$ Engineering college, China JiLiang University, Hangzhou China \\ ${ }^{1}$ lou999@gmail.com, ${ }^{2}$ wuchen0715@163.com
}

\begin{abstract}
Federal Kalman filter is often used in multi-sensor wireless localization system. Int this paper, we estimate the target location in non line of sight (NLOS) propagation Ap approach based on federal Kalman filter is proposed, which is called smooth-federal Kalman filter. Federal Kalman filter uses standard Kalman filter in local filter to processes data in a forward recursion. Kalman smoother which processes data in a backwatrd recursion is addressed in ordinary federal Kalman filter. Simulation and comparison results show that the estimation of the proposed approach performs superior to fedenal Kalman filter in NLOS propagation.
\end{abstract}

Keywords: wireless localization; non line of sight (NLOS), federal Kalman filter; Kalman filter; Kalman smoother

\section{Introduction}

Wireless localization is achieved by using measurements from target to anchors. In general, localization can be obtained by using (ime of arrival (TOA) measurements [1-2], time difference of arrival (TDOA) measurements [3-4], angle of arrival (AOA) measurements [5-6], received signal strength (RSS) measurements [7-8], or a combination of above [9]. In this paper we consider using TDOA measurements.

R. E. Kalman first introduced Kalman filter [10] in his paper in 1960. Kalman filter uses priori information to obtain prediction information in the first step. And then, Kalman filter processes measurement information and prediction information in update step. Kalman smoother is a smoother which can reduce divergence and improve the accuracy of a filter. The difference between Kalman filter and Kalman smoother is that the recursion in Kalman filter is forward but back ward in Kalman smoother. Kalman smoother is introduced by Rauch, Tung and Striebel in 1965 [11], so it is also named RTS smoother.

Based on Kalman filter, people utilized information fusion technique to produce centralized Kalman filter [12] and decentralized Kalman filter [13]. In 1998, N. A. Carlson [14-15] proposed federal Kalman filter on the basis of decentralized Kalman filter. Federal Kalman iliter applies information allocation technique, besides it consists of a master filter and several local filters. It is should be pointed out that our algorithm is based on [16].

This paper focuses on the improvement of federal Kalman filter in wireless localization. The rest of the paper is organized as follows. Section 2 describes federal Kalman filter architecture. In Section 3, we improve the performance of federal Kalman filter by using Kalman smoother. Simulation results are presented in Section 4 in order to evaluate the performance of the improvement based on federal Kalman filter. Finally, conclusions are drawn in Section 5. 


\section{Federal Kalman Filter Architecture}

Federal Kalman filter is a special kind of decentralized Kalman filter, which is made up of a master filter and a couple of local filters. In fact, we adapt standard Kalman filters as local filters in the first stage. It's noteworthy that, by using noise variance upper bound technique, each local filter is independent with each other. The structure of local filters is decentralized and parallel. Moreover, each local filter is connected with a specific sensor and a reference sensor. Time update and measurement update are carried out in all local filters, resulting in producing suboptimal estimates. Master filter fuses those suboptimal estimates from local filters, thus, global optimal estimation is obtained. More importantly, master filter sends global optimal estimate back to each local filter to form an iterative procedure, including information allocation technique. Figure 1 shows the architecture of federal Kalman filter. According to the strategy of information allocation, federal Kalman filter can be divided into four modes, corresponding to four architectures.

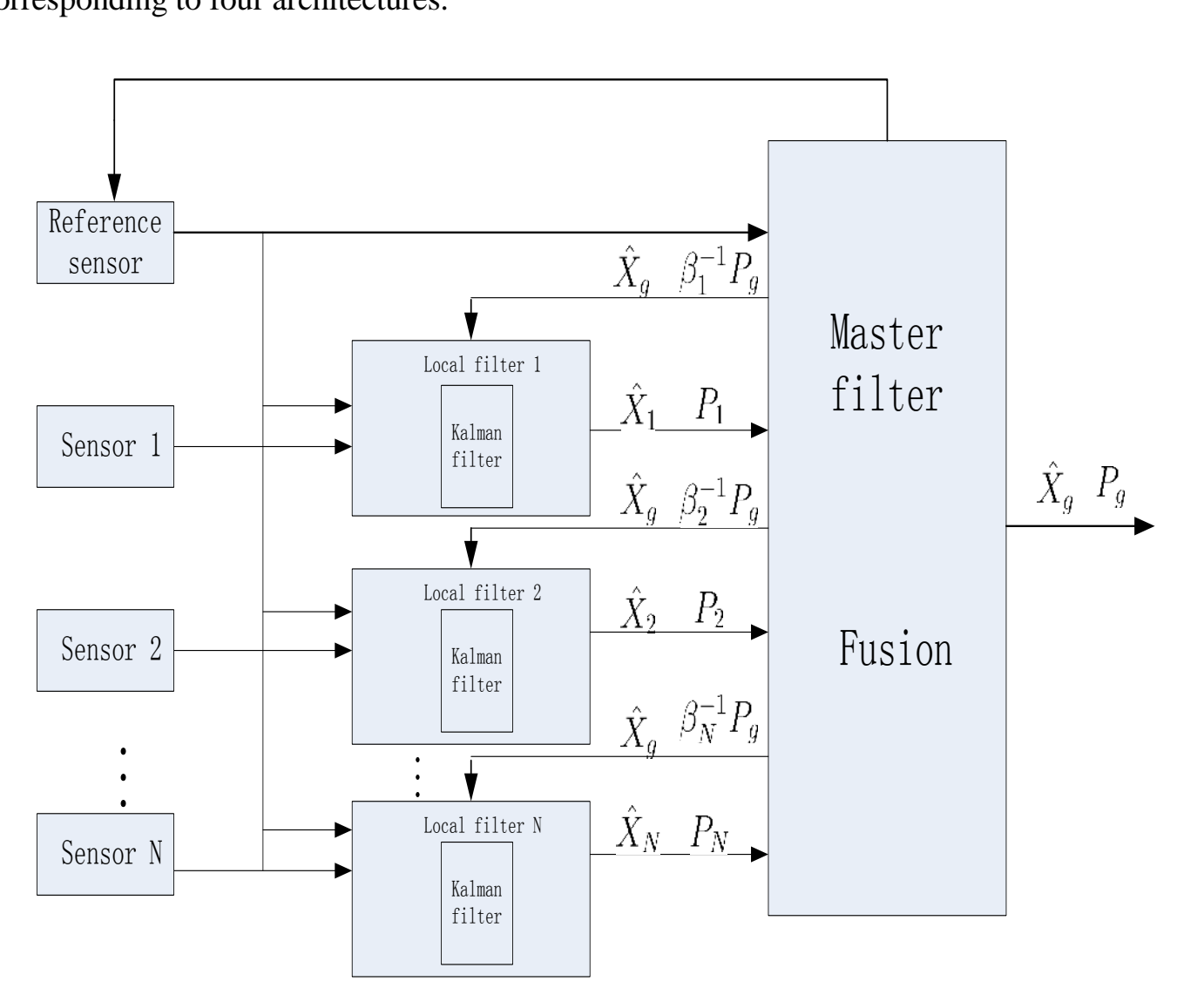

Figure 1. The Structure of Federal Kalman Filter

\section{Algorithm based on Federal Kalman Filter}

\subsection{NLOS Alleviation}

NLOS propagation generates from obstacles between target and anchor, which result in large scale error in TDOA measurements. Since TDOA measurements are not accuracy, the performance of federal Kalman filter in localization would be severely impaired. Then, Kalman filter is considered in the first place to reduce NLOS bias through optimal estimation. The algorithm of Kalman filter has two steps: In the prediction step, the priori information is used to predict the next state of the system.

$$
\begin{aligned}
& X(k)=A X(k-1)+B U(k) \\
& P(k)=A P(k-1) A^{T}+Q
\end{aligned}
$$


The update step estimates the current state of the system.

$$
\begin{gathered}
X(k)=X(k-1)+K_{g}(k) \cdot(Z(k)-H X(k-1)) \\
K_{g}(k)=P(k-1) H^{T}\left(H \cdot P(k-1) \cdot H^{T}+R\right)^{-1} \\
P(k)=\left(I-K_{g}(k) \cdot H\right) \cdot P(k-1)
\end{gathered}
$$

The specific process of Kalman filter can be read in [10]. By using Kalman filter optimal TDOA measurements are obtained, which turns out to be more precise than originals.

\subsection{Information Allocation}

Subsystem is made up of a reference sensor and some common sensors. Each sensor estimates the target location by MMSEE [17] algorithm in different environment. Note that target location obtained by reference sensor should be more reliable than other sensors. It usually obtains from master filter after initialization. Local filter $i$ processes output from reference sensor and output from sensor $i$.

$$
X_{i}=\left[\begin{array}{c}
X_{R} \\
X_{s i}
\end{array}\right]
$$

Where $X_{i}$ denotes the estimate from local filter $i . X_{R}$ denotes the estimate from reference sensor processed by local filter $i$. $X_{i}$ denotes the estimate from sensor $i$ processed by local filter $i$.

We should give the initial value in the first place. Initial suboptimal covariance $P_{i}$ is obtained from local filters. Then initial optimal covariance $P_{g}$ is obtained by (14).

After initialization, optimal covariance $\mathcal{P}_{g}$ is alfocated to master filter and all local filters.

$$
P_{i} Z_{i}^{-1} P_{g}, \quad \dot{c}=1 \ldots \mathrm{N}, m
$$

$\beta_{i}$ is the local filter information allocanon coefficient, $\beta_{m}$ is the master filter information allocation coefficient. An 1 is the sum of local filters. $\beta_{i}$ and $\beta_{m}$ meet the information allocation principle

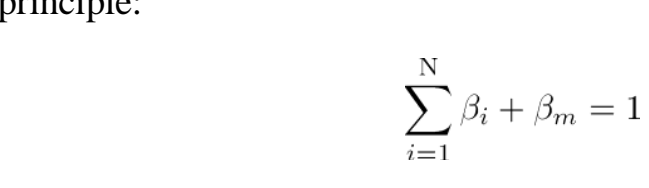

Allocation coefficient is usually calculated by $\beta_{i}=\beta_{m}=\frac{1}{\mathrm{~N}+1}$. It is easy to understand that the high accuracy the local filter has, the larger allocation coefficient should be. Besides, covariance shows the estimate accuracy of a local filter. Therefore, we calculate allocation coefficient as follows.

$$
2 \beta_{i}=\frac{P_{i}}{\sum_{i=1}^{\mathrm{N}, m} P_{i}}
$$

\subsection{Measurement Update}

Measurement update occurs only in local filters.

$$
P_{i}(k)=P_{i}(k-1)-P_{i}(k-1) H_{i} A_{i}^{-1} P_{i}(k-1)
$$

Where $A_{i}=H_{i} P_{i}(k) H_{i}^{T}+R_{i}, P_{i}$ denotes the covariance of local filter $i, R_{i}$ denotes measurement covariance, $I_{i}$ denotes measurement matrix. 


\subsection{Time Update}

Master filter and local filters execute time update independently.

$$
\begin{aligned}
& X(k)=\Phi X(k-1)+\Gamma U \\
& P(k)=\Phi P(k-1) \Phi^{T}+Q
\end{aligned}
$$

Where $\Phi$ represents state shift matrix, $X(k)$ represents the estimate of target, $\Gamma$ represents system noise matrix, $U$ represents system control matrix, $Q$ represents system covariance matrix.

\subsection{Global Optimal Fusion}

Suboptimal estimate $X_{i}$ and suboptimal covariance $P_{i}$ from local filters are sent to master filter. Global optimal estimate $X_{g}$ and global optimal covariance $P_{g}$ are achieved by applying information fusion technique.

$$
\begin{aligned}
& X_{g}=P_{g} \sum_{i=1}^{N, m} P_{i}^{-1} X_{i} \\
& P_{g}^{-1}=\sum_{i=1}^{N, m} P_{i}^{-1}
\end{aligned}
$$

\subsection{Improvement by Kalman Smoother}

Federal Kalman filter applies Kalman niter proces ing data forward going from the first measurement towards the last one in each local fitten However, we use a Kalman smoother which processes data in the opposite direction after a Kalman filter in each local filter. This algorithm begins with the forward filtered estimate. The recursion procedure of Kalman smoother is given below.

$$
\begin{gathered}
X(k+1)=A X(k)+B U(k) \\
P(k+1)=A P(k) A^{T}+Q \\
C(k) \quad P(k) A^{T} P(k+1)^{-1} \\
X^{s}(k)=X(k)+C(k)\left(X^{s}(k+1)-X(k+1)\right) \\
P^{s}(k)=P(k)+C(k)\left(P^{s}(k+1)-P(k+1)\right) C(k)^{T}
\end{gathered}
$$

Where $X^{s}(k)$ is the Kalman smoother estimate, $P^{s}(k)$ is the Kalman smoother estimate covariance, $X(k)$ is the Kalman filter estimate, $P(k)$ is the Kalman filter estimate covariance, $C(k)$ is the Kalman smoother gain. Figure 2 shows the new structure of federal Kalman filter. 


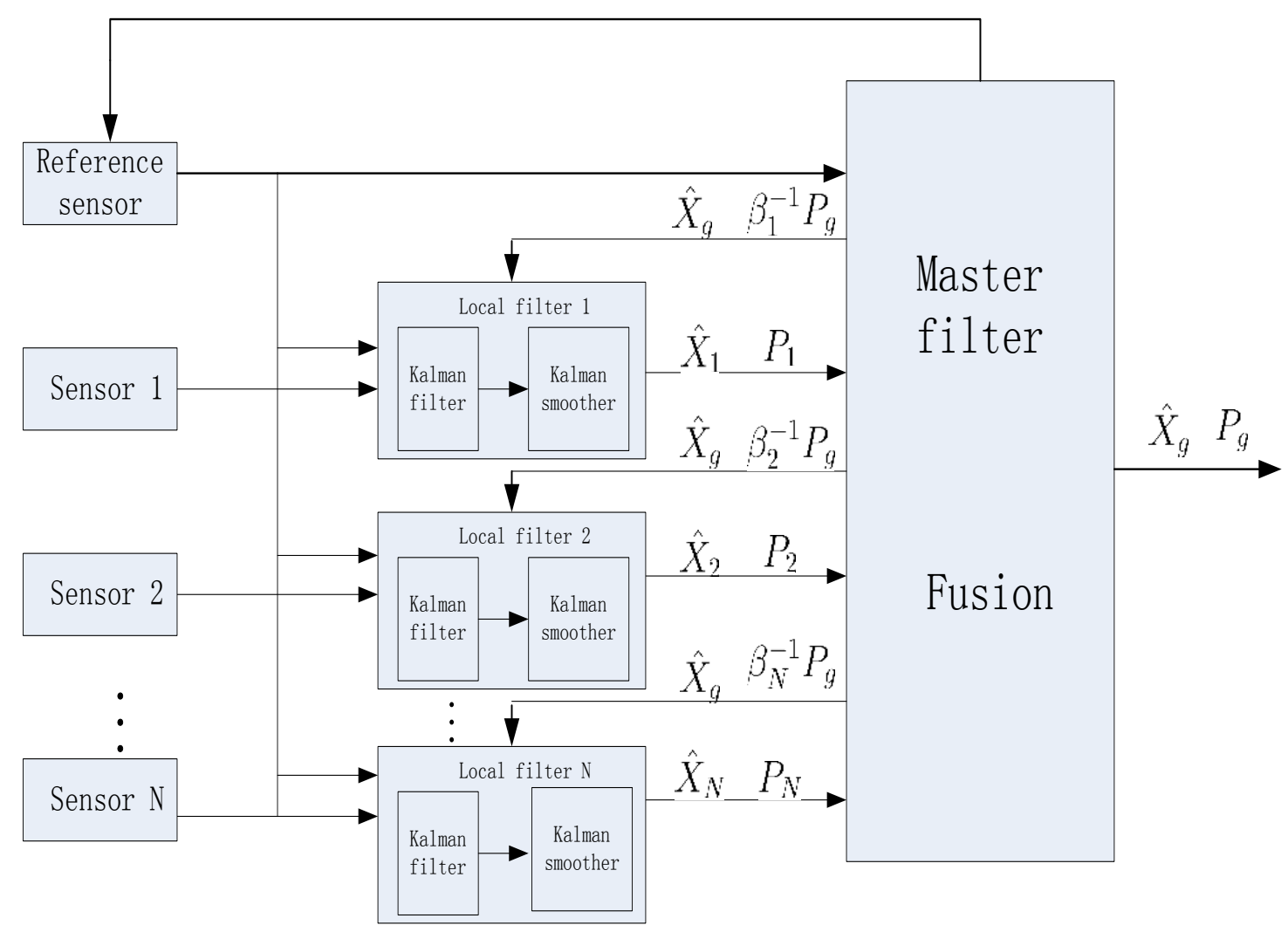

Figure 2. The Structure of Smooth-federal Kalman Filter

\section{Simulations}

We conduct our simulations in NLOS propagation. In NLOS propagation, TDOA model can be written as

$$
2+t_{i}-t_{1}+\mathrm{NLOS}_{i 1}, \quad i=2, \ldots, \mathrm{N}
$$

Where $t_{i}$ denotes the propagation time from target to anchor $i, n_{i 1}$ denotes noise. $\operatorname{NLOS}_{i 1}$ denotes the NEOS bias between anchor $i$ and anchor 1 . The relationship between anchor $i$ and anchor 1 can be written as

$$
r_{i 1}=r_{1}+c * n_{i 1}+c * \mathrm{NLOS}_{i 1}, \quad i=2, \ldots, \mathrm{N}
$$

Where $r_{i}=c * t_{i}, r_{i 1}=\left(t_{i}-t_{1}\right), r_{i 1}$ denotes the range difference between anchor $i$ and anchor $1, r_{i}$ is the true distance between anchor $i$ and target, $c$ denotes signal propagation velocity.

We simulate three $(\mathrm{N}=3)$ different noises $n_{i, 1}$ to produce three environments. Then, each sensor (including a reference sensor) estimates the target location in an environment, respectively) Target is supposed to run by $1.4 \mathrm{~m} / \mathrm{s}$ in the system where lays four $(\mathrm{M}=4)$ distributed anchors. Four anchors locate at $[18,85],[90,90],[30,25]$ and $[100,8]$, respectively. All results are averages of 50000 independent runs. The performance of the proposed approach is evaluated in terms of standard deviation and mean error.

In the first stage of simulation we show the NLOS alleviation by using Kalman filter. In figure 3, the green dots represent the real range difference between anchor 1 and anchor 2, the red dots represent the measured range difference in NLOS propagation, and the blue dots represent the measured range difference processed by Kalman filter. It's obvious that Kalman filter has a remarkable effect on NLOS bias. 


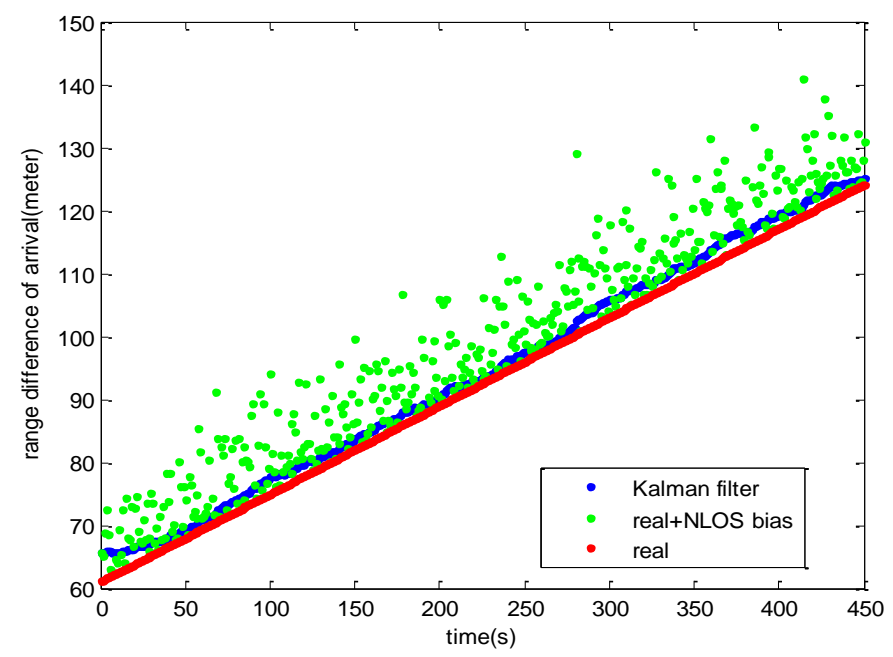

Figure 3. Kalman Filter on Reducing NLOS Bias

Figure 4 gives the velocity comparison curves of smooth-federal Kalman filter and federal Kalman filter, where red dot is the real velocity of target, blue dot is the target velocity processed smooth-federal Kalman filter, green dot is the target velocity processed federal Kalman filter. Thus smooth-federal Kalman filter has a good yelocity estimation than federal Kalman filter.

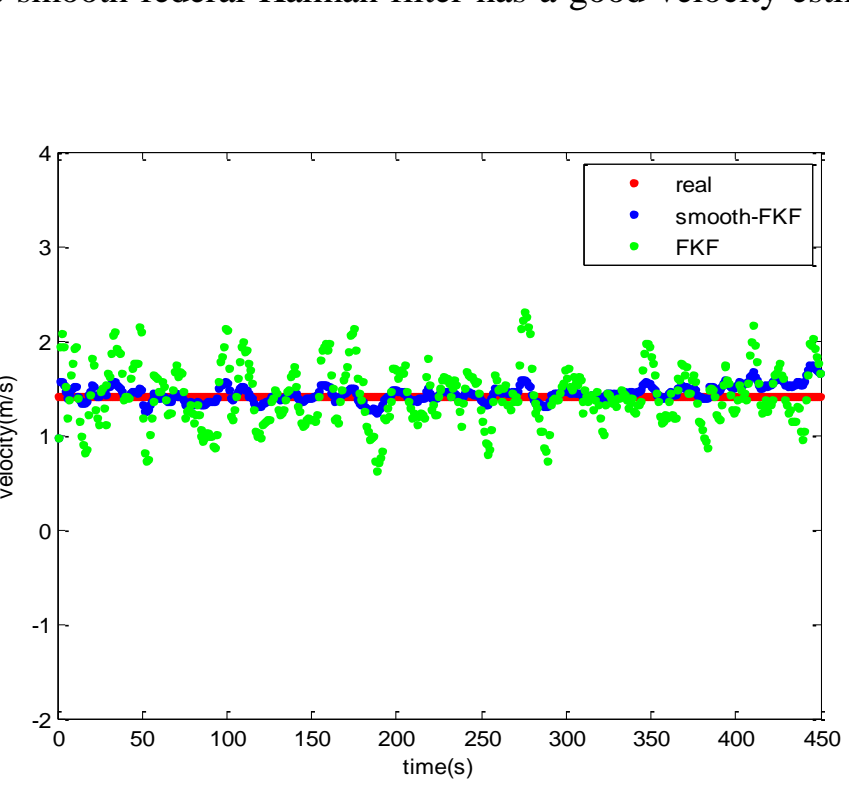

Figure 4. Velocity by Smooth-federal Kalman Filter

Figure 5 shows standard deviation against NLOS bias for both two approaches. The standard deviation of the smooth-federal Kalman filter is smaller than federal Kalman filter as NLOS bias increases. And the gap between the smooth-federal Kalman filter and federal Kalman filter is becoming larger when NLOS bias turns larger. In this case, the proposed approach outperforms federal Kalman filter in different NLOS propagation. As a matter of fact, standard deviation shows the location stability capability. Thus, we can conclude that the localization of the smooth-federal Kalman filter is more stable when NLOS environment is more serious. From this view of point, smooth-federal Kalman filter is more stable than federal Kalman filter. 


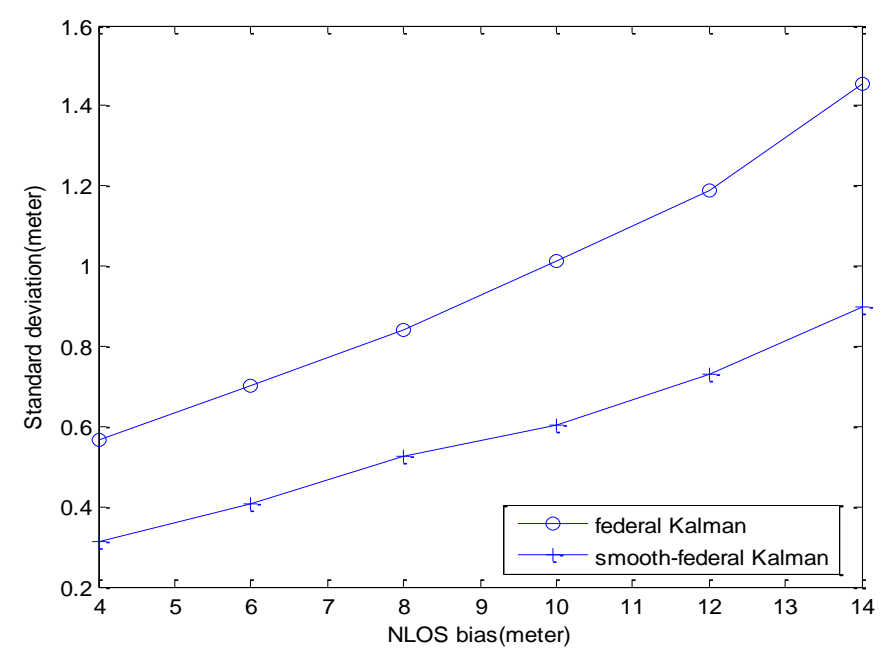

Figure 5. Standard Deviation against NLOS Bias

Mean error is used to evaluate the precision of localization No matter how NLOS bias changes, the mean error of smooth-federal Kalman fitter is smaller than federal Kalman filter in Figure 6, which points to the fact that the location accuracy of smooth-federal Kalman filter is better than federal Kalman filter.

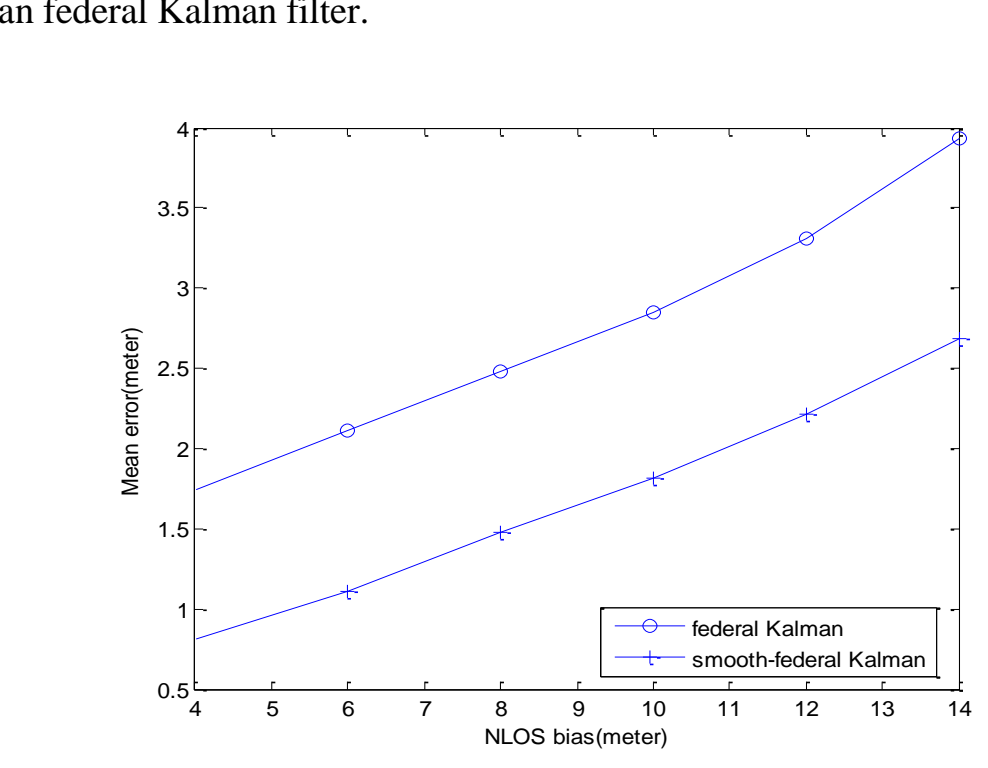

Figure 6. Mean Error against NLOS Bias

Table 1 and Table 2 show the standard deviation and mean error of smooth-federal Kalman filter and federal Kalman filter when reference sensor uses different target location. We can also draw the same conclusion that smooth-federal Kalman filter performs better than federal Kalman filter from Table 1 and Table 2.

Table 1. Standard Deviation Comparison

\begin{tabular}{|c|c|c|c|c|c|c|}
\hline NLOS bias(meter) & 4 & 6 & 8 & 10 & 12 & 14 \\
\hline $\begin{array}{c}\text { smooth-federal } \\
\text { Kalman(meter) }\end{array}$ & 0.4341 & 0.5829 & 0.7643 & 0.9498 & 1.1458 & 1.3979 \\
\hline $\begin{array}{c}\text { federal } \\
\text { Kalman(meter) }\end{array}$ & 0.7569 & 0.9801 & 1.1685 & 1.4082 & 1.7076 & 2.0013 \\
\hline
\end{tabular}


Table 2. Mean Error Comparison

\begin{tabular}{|c|c|c|c|c|c|c|}
\hline $\begin{array}{c}\text { NLOS } \\
\text { bias(meter) }\end{array}$ & 4 & 6 & 8 & 10 & 12 & 14 \\
\hline $\begin{array}{c}\text { smooth-federal } \\
\text { Kalman(meter) }\end{array}$ & 0.8824 & 1.2719 & 1.5936 & 1.8945 & 2.2129 & 2.7802 \\
\hline $\begin{array}{c}\text { federal } \\
\text { Kalman(meter) }\end{array}$ & 1.8851 & 2.2396 & 2.6032 & 2.9849 & 3.4484 & 4.1331 \\
\hline
\end{tabular}

\section{Conclusion}

In wireless localization, NLOS propagation is a dominant source of location errors. It usually exists in indoor environment or urban environment. In this paper, we focus mitigating NLOS propagation by addressing smooth-federal Kalman filter. The simulation results have proved that by using smooth-federal Kalman filter a good localization performance in NLOS propagation can be achieved.

\section{References}

[1] E. Xu, Z. Ding and S. Dasgupta, "Source Localization in Wireless Sensor Networks from Signal Time-of-arrival Measurements", IEEE Trans. Signal Process, ol. 59, no. 6, (2011) June, pp. 2887-2897.

[2] H. Shen, Z. Ding, S. Dasgupta and C. Zhao, "Multiple Source Localization in Wireless Sensor Networks Based on Time of Arrival Measurement", IEEE Trans. Signal "Process, vol. 62, no. 8, (2014) April, pp. 1938-1949.

[3] Y. Huang, J. Benesty, G. W. Elko, and R. Mersereau, "Real-tme Passive Source localization: a practical linear-correction least-squares approach" IEEE Trans. Speech Audio Process, vol. 9, no. 8, (2001) November, pp. 943-956.

[4] S. Zhong, W. Xia and Z. He, "Adaptive Direct Position Determination of Emitters Based on Time Differences of Arrival, Signal and Information Processing”, IEEE China Summit \& International Conference on Beijing, China, (2013) July 6-10, pp. 230-234.

[5] R. O. Schmidt, "Multiple Emitter Location and Signal Parameter Estimation", IEEE Trans. Antennas Propag, vol. 34, no. 3, (1986) March, pp. 276-280.

[6] C. Wang, F. Qi, G. Shi and X. Wang, "Convex Combination Based Target Localization with Noisy Angle of Arrival Measurement,", IEEE Wireless Communications Letters, vol. 3, no.1, (2014) February, pp.14-17.

[7] A. O. Hero III, M. Perkins, N. S. Correal, and R. J. O' Dea, "Relative Location Estimation in Wireless Sensor Networks", TEEE Trans. Signal Process, vol. 51, no. 8, (2003) August, pp. 2137-2148.

[8] H. Zhang and X. Shi, "ANew/ Thdoor Localization Technology Using Back Propagation Neural Network to Fit the RSSI-d Curve, Intelligent Control and Automation”, 10th World Congress on Beijing, China, (2012) July 6-8, pp. 80-83.

[9] J. Chen and A. Abeci "A Hybrid Framework for Radio Localization in Broadband Wireless Systems", IEEE Global Telecomnunications Conference, Miami, America, (2010) December 6-10, pp. 1-6.

[10] R. E. Kalman, "A New Approach to Linear Filtering and Prediction Problems", Transactions of the ASME Journal of Basic Engineering, vol. 83, (1960), pp. 95-107.

[11] H. E. Rauch, F. Tung and C. T. Striebel, "Maximum Likelihood Estimates of Linear Dynamic Systems", AIA Journal, vol. 3, no. 8, (1965) August, pp. 1445-1450.

[12] J. Sijs, M. Lazar, P. Van de Bosch and Z. Papp, "An Overview of Non-centralized Kalman Filters, IEEE Conference on. Conference on Control Applications, (2008) September 3-5, San Antonio, America.

133 H. Hashemipour, S. Roy and A. Laub, "Decentralized Structures for Parallel Kalman Filtering”, IEEE Trans. Automat. Contr, vol. 33, no. 1, (1988) January, pp. 88-94.

[14] N. A. Carlson, "Federated Square Root Filter for Decentralized Parallel Processes", IEEE Trans. Aerosp. Electron. Syst, vol. 26, no. 3, (1990) May, pp. 517-525.

[15] N. A. Carlson and M. P. Berarducci, "Federated Kalman Filter Simulation Results", Navigation, Journal of the Institute of Navigation, vol. 41, no. 3, (1994), pp. 297-321.

[16] M. P. Wylie and J. Holtzman, "The Non-Line of Sight Problem in Mobile Location Estimation", Universal Personal Communications, 5th IEEE International Conference on Cambridge, MA, America, vol. 2, (1996) September 29-October 2, pp. 827-831.

[17] J. Y. Huang, Q. Wan and P. Wang, "Minimum Mean Square Error Estimator for Location Using Time-difference-of-arrival Measurements", IET Radar Sonar Navigation, vol. 5, (2011) February, pp. 137-143. 


\section{Authors}

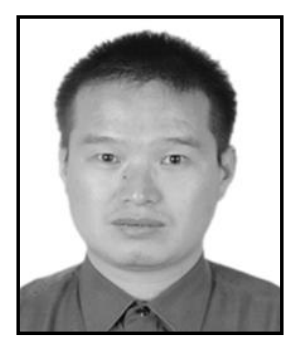

\section{Xi Zhong Lou}

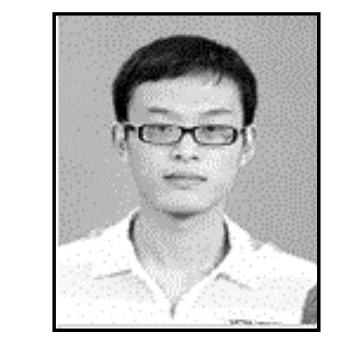

Dao Rong Wu

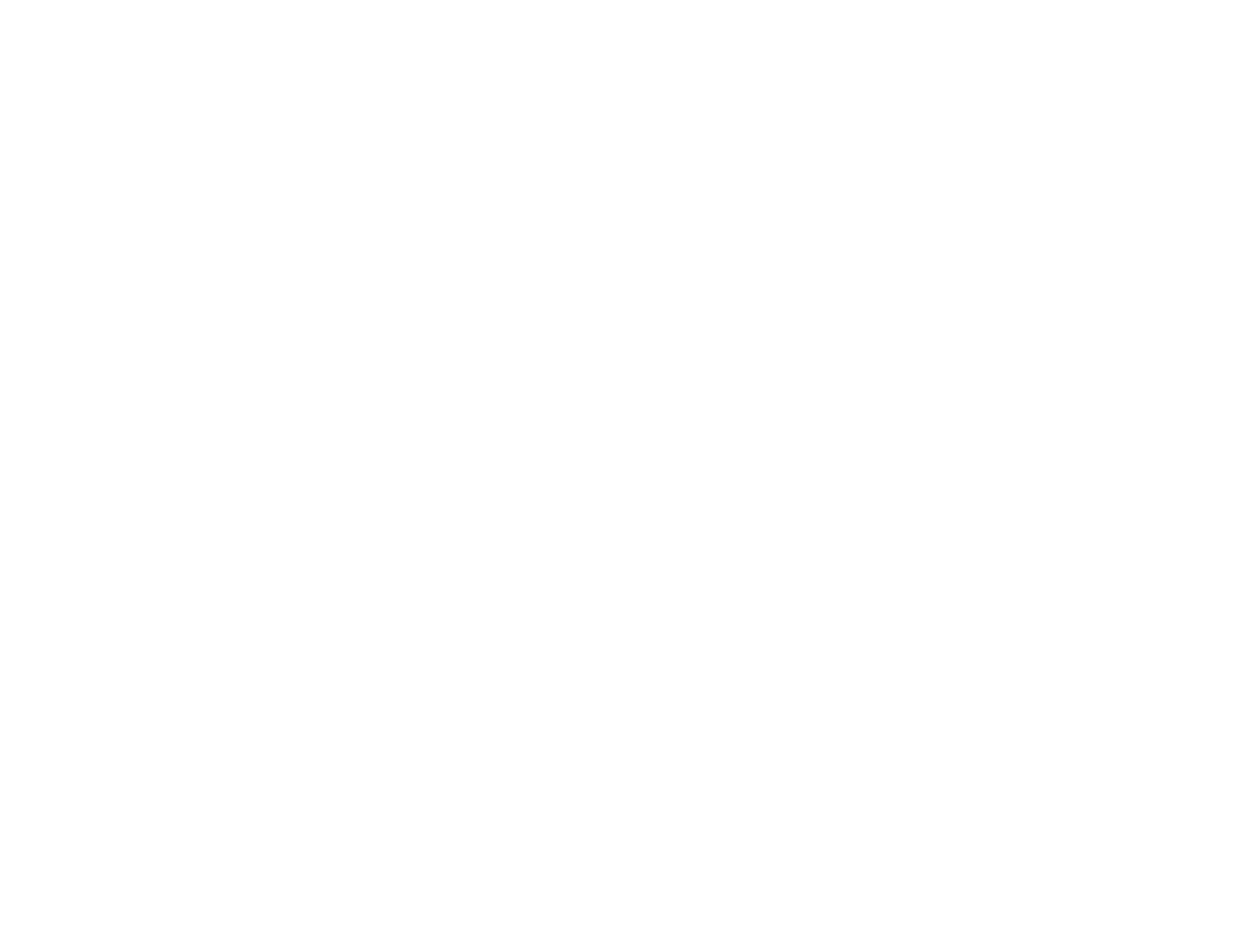




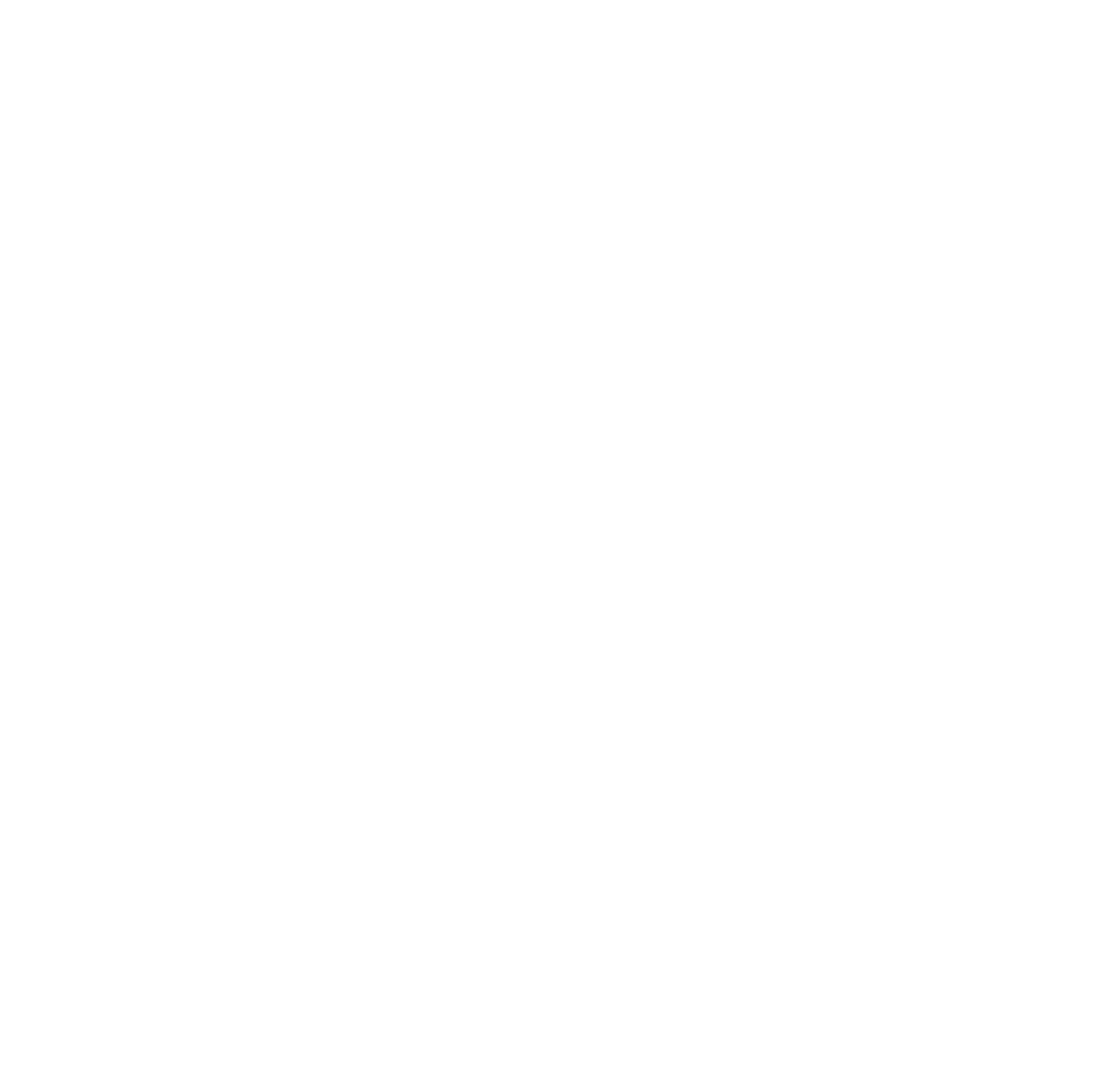

